\title{
Oral surgical procedures under local anaesthesia in day surgery
}

\author{
Abdulkadir Burak Çankaya, Çağrı Akçay ${ }^{*}$ Neşe Kahraman and Banu Gürkan Köseoğlu
}

\begin{abstract}
Background: The objective of this study was to analyze gender-stratified data of patients who underwent day surgery in a hospital based on the type of treatment, type of local anaesthesia, and local anaesthesia complications. By learning all these parameters, it is our main goal to find answers to questions such as what we can do in hospital conditions, what we can win, and what operations we can treat.

Methods: A retrospective review was performed to assess hospital records of 10,750 dental patients who received oral surgery under local anaesthesia at the Istanbul University Department of Oral and Maxillofacial Surgery from August 2013 through June 2016.

Results: Patients mostly received surgery for wisdom teeth, dental implants, or odontogenic cysts or tumours. Men aged 31-40 years (23.66\%) and women aged 21-30 years (30.73\%) were the largest groups undergoing operations. Surgery for an impacted tooth was the most common ambulatory procedure, accounting for $54.2 \%$ of operations. The second most common ambulatory procedure was dental implant surgery (10.2\%), followed by root (7.4\%), odontogenic cyst (7.2\%), and impacted canine surgeries (6.4\%). The most common age group receiving surgery was 21-30 years old (3304 patients, 60.75\%). Twice as many women as men underwent surgery.

Conclusions: Day case surgery is an expanding area of health care and a valuable method of treating patients in many aspects of oral surgical practice. Different medical and dental specialties can benefit from this ambulatory approach to treatment, which also reduces treatment costs.
\end{abstract}

Keywords: Day case surgery, Local anaesthesia, Gender, Type of treatment

\section{Background}

Local anesthesia results from the prevention of the spread of the action potential so that the sensation cannot be transferred from the stimulus source, such as periodontium and tooth, to the brain. Sodium ions are necessary for a potential action to occur in associated nerve membrane. Local anesthetics work by blocking the entry of sodium ions into their channels. As a result, temporary increase in the permeability of the nerve membrane is prevented [1].

In 1996, Marin described the ambulatory surgery term as an optimal organizational model for multidisciplinary surgical care that permits effective and safe treatment of some patients without having to resort to conventional hospital beds. This concept involves interventions of

* Correspondence: cgrakcay@gmail.com

Faculty of Dentistry, Oral and Maxillofacial Surgery, İstanbul Üniversitesi, Istanbul, Turkey

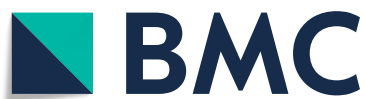

moderate complexity, performed under general, local or regional anesthesia, which do not require hospitalization after surgery [2].

Day surgery is an expanding field of medical care and an invaluable method for treating patients in many aspects of oral surgery practice. Several medical and dental specialties can benefit from this outpatient treatment approach. In oral surgery, the accepted daily procedure includes tooth extraction, root fragment extraction, orthodontic dental fenestration, removal of small cystic lesions, hard and soft tissue biopsies and frenectomy, extraction of osteosynthesis plaques and wires from the jaws, soft tissue surgery, laser surgery and cryosurgery [3].

A retrospective study was performed to analyze medical records of patients who had been treated at the Istanbul University Department of Maxillofacial and Oral Surgery during the period from January 2010 to

(C) The Author(s). 2018 Open Access This article is distributed under the terms of the Creative Commons Attribution 4.0 International License (http://creativecommons.org/licenses/by/4.0/), which permits unrestricted use, distribution, and reproduction in any medium, provided you give appropriate credit to the original author(s) and the source, provide a link to the Creative Commons license, and indicate if changes were made. The Creative Commons Public Domain Dedication waiver (http://creativecommons.org/publicdomain/zero/1.0/) applies to the data made available in this article, unless otherwise stated. 
July 2013. The aim of this study was to gain insight into the types and frequencies of diagnoses and ambulatory surgical treatments at the Department of Oral Surgery in Istanbul University. We also explored whether there was a significant difference in procedures according to sex, age, and operation side. By learning all these parameters, it is our main goal to find answers to questions such as what we can do in hospital conditions, what we can win, and what operations we can treat.

\section{Methods}

A total of 10,750 patients admitted to the Istanbul University Department of Oral Surgery from 2010 January to 2013 July were included in the study. The data were collected retrospectively from ambulatory outpatient operation protocols. The data was collected from clinic protocol records. The form for the ambulatory clinic protocols classified the patients according to date of arrival, age, sex, place of residence, and surgical treatments. After data collection, surgical treatments were categorized to facilitate statistical analysis.

Statistical calculations were performed with Number Cruncher Statistical System 2007 Statistical Software (Utah, USA) for Windows. Besides standard descriptive statistical calculations (mean and standard deviation, frequency, percentage), one-way ANOVA was used to compare multiple groups, the post hoc Tukey's multiple-comparison test was used to compare subgroups, unpaired t-tests were used to compare two groups, and the Chi square test was performed to evaluate qualitative data. Statistical significance was established at $p<0.05$.

\section{Results}

A total of 10,750 dental patients were evaluated. This study included 4126 (38.38\%) men and 6624 (61.62\%) women, whose mean age was 37.70 years. Men aged 3140 years (23.66\%) and women aged 21-30 years $(30.73 \%)$ were most common in this data set (Table 1).

The 21-30-year age group was the largest, with 3304 patients $(60.75 \%)$. The patient number in the group aged $<10$ years was low because paediatric surgery generally employs general anaesthesia (Table 2). For both men and women, the most common surgery was for an impacted third molar (49.93 and 56.79\%, respectively). On the other hand, cyst operations were more prevalent in men $(10.18 \%)$ than in women $(5.39 \%)$ (Table 3$)$.

Table 1 Total patient and gender distribution

\begin{tabular}{llllll}
\hline & N & Mean & SD & Min & Max \\
\hline Men & 4126 & 38.17 & 13.47 & 8 & 90 \\
Women & 6624 & 37.41 & 13.32 & 7 & 86 \\
Total & 10,750 & 37.70 & 13.39 & 7 & 90 \\
\hline
\end{tabular}

Table 2 Age distribution

\begin{tabular}{lllll}
\hline Age (years) & Men $(n)$ & Men $(\%)$ & Women $(n)$ & Women $(\%)$ \\
\hline$<10$ & 5 & 0.12 & 6 & 0.09 \\
$11-20$ & 301 & 7.30 & 525 & 7.93 \\
$21-30$ & 1189 & 28.82 & 2115 & 31.93 \\
$31-40$ & 1010 & 24.48 & 1533 & 23.14 \\
$41-50$ & 863 & 20.92 & 1325 & 20.0 \\
$51-60$ & 472 & 11.44 & 691 & 10.43 \\
$61-70$ & 214 & 5.19 & 330 & 4.98 \\
$>71$ & 72 & 1.75 & 99 & 1.49 \\
\hline
\end{tabular}

This study included surgical treatments in the ambulatory clinic and ambulatory operation room. Impacted tooth operations were the most common ambulatory procedure at $54.2 \%$. The second most common ambulatory procedure was dental implantation (10.2\%), followed by root (7.4\%), odontogenic cyst (7.2\%), and impacted canine operations (6.4\%). (Table 4).

The third molar was the most common impacted tooth, with a rate of $54.2 \%$, followed by canines $(6.4 \%)$ and premolars (0.7\%) (Table 5).

Operations most commonly involved the mandibular arch (63.16\%) (Table 6).

The posterior region was the most common surgical region for both mandible and maxilla (75.92\%); posterior surgeries were nearly four times as common as those in the anterior region $(22.49 \%)$ (Table 7).

\section{Discussion}

The potential advantages of day surgery include:

1. Patient benefits: Day surgery results in reduced waiting time and shortened hospital stay, leading to reduced risk of nosocomial infections, less interference with daily life activities, and the possibility of choosing from among various treatment alternatives.

2. Reduced costs: Day case surgery is more costeffective than surgery with patient hospitalization. Savings in services are obtained, although the actual surgical costs are very similar in both modalities.

3. Improved quality patient care: Day case surgery can be performed with the same or even greater health care quality than surgery involving patient hospitalization. Previous studies showed that day surgery leads to better results than surgery with hospitalization, with fewer and less serious postoperative complications and a lower readmission rate. 
Table 3 Operation types in genders

\begin{tabular}{|c|c|c|c|c|c|}
\hline Operation type & Men $(n)$ & Men (\%) & Women $(n)$ & Women (\%) & $p$ \\
\hline Impacted third molar & 2060 & 49.93 & 3762 & 56.79 & 0.0001 \\
\hline Implant & 429 & 10.40 & 669 & 10.10 & 0.62 \\
\hline Root & 352 & 8.53 & 445 & 6.72 & 0.0001 \\
\hline Odontogenic cyst & 420 & 10.18 & 357 & 5.39 & 0.0001 \\
\hline Impacted canine & 230 & 5.57 & 454 & 6.85 & 0.008 \\
\hline Apical resection & 239 & 5.79 & 333 & 5.03 & 0.086 \\
\hline Alveoloplasty & 112 & 2.71 & 134 & 2.02 & 0.02 \\
\hline Vestibuloplasty & 83 & 2.01 & 156 & 2.36 & 0.24 \\
\hline Odontogenic tumour & 38 & 0.92 & 62 & 0.94 & 0.937 \\
\hline Frenectomy & 37 & 0.90 & 44 & 0.66 & 0.175 \\
\hline Impacted premolar & 30 & 0.73 & 42 & 0.63 & 0.565 \\
\hline
\end{tabular}

4. Shortened waiting lists: The need for waiting lists is a complex phenomenon that cannot always be solved by increasing available health care resources [3].

Records of 10,750 patients treated in the oral ambulatory operation room were examined in this study. Surgically treated patients in a study by Cabov et al. had an age range of 5-88 years, with a median age of 37 years for men and 31 for women. The numbers in the present study did not differ significantly from other reports. Our patients had a similar age range, although women had a mean age of 37.

Syed et al. showed that in both males and females, impacted third molars were more prevalent in the mandibular arch than in the maxilla (49.5\% vs. $48.62 \%)$. In that study, the male-to-female ratio for impacted third molars was 604: 109 (5.54: $1 ; p=0.707$ ). In our study, this surgery was also more common in the mandibular arch (63.16\%), and the male-to-female ratio for the impacted third molar operation was 2060: 3762 (49.93, 56.79; $p=0.0001)$. According to Syed et al., the 2025-year age group showed the highest prevalence of third molar impaction (64.5\%), and the frequency decreased

Table 4 Operation types

\begin{tabular}{lll}
\hline Operation type & Number & $\%$ \\
\hline Impacted third molar & 5822 & 54.2 \\
Implant & 1098 & 10.2 \\
Root & 797 & 7.4 \\
Odontogenic cyst & 777 & 7.2 \\
Impacted canine & 684 & 6.4 \\
Apical resection & 572 & 5.3 \\
Alveoloplasty & 246 & 2.3 \\
Vestibuloplasty & 239 & 2.2 \\
Odontogenic tumour & 100 & 0.9 \\
Frenectomy & 81 & 0.8 \\
\hline
\end{tabular}

with increasing age. Similarly, in our study, the 21-30-year age group most commonly underwent this procedure, with 3304 patients $(60.75 \%)$. In other studies, as in ours, the prevalence of impacted third molars was much higher in the mandibular than in the maxillary arch $(49.4 \%$ vs. 18.4\%) [4].

In the day surgery, the most common oral surgery routine is the extraction of the third molar. In a literature review performed by Ruiz-Mirete and Gay-Escoda, the lower thirds of the mandible were the most commonly impacted teeth [3]. Surgical removal of impacted teeth is most common procedure for several reasons:

1. Reducing in the caries index implies fewer extractions of non-impacted teeth. This may partly explain the greater extraction of impacted teeth, including third molars.

2. The increase in demand for orthodontic treatment has, in turn, increased the number of impacted teeth operations (extraction or fenestration of permanent upper canines) or the extraction of other permanent teeth, such as premolars.

3. The number of panoramic radiography devices have increased in the last 25 years. This additional radiographic technique allows for early detection of impacted teeth as well as control of eruption turnover and identification of dental and/or maxillary pathology requiring surgery [3].

In our study, more women than men underwent surgery, in accordance with previous findings. This

Table $\mathbf{5}$ Impacted teeth numbers

\begin{tabular}{lll}
\hline Impacted teeth & Number & $\%$ \\
\hline Third molar & 5822 & 54.2 \\
Canine & 684 & 6.4 \\
Premolar & 72 & 0.7 \\
\hline
\end{tabular}


Table 6 Operated jaw numbers

\begin{tabular}{lll}
\hline Operated jaw & Number & $\%$ \\
\hline Maxilla & 3676 & 34.20 \\
Mandible & 6790 & 63.16 \\
Maxilla and mandible & 250 & 2.33 \\
Temporomandibular joint (TMJ) & 34 & 0.32 \\
\hline
\end{tabular}

could reflect increased prevalence of caries among women. In addition, this may be related to women's increased interest in aesthetics and health care. Women and men had more operations for impacted wisdom tooth and implants in other countries A study in North India also found that women had more surgeries for impacted teeth more than men [5]. In our study, the number of operations for impacted wisdom teeth was 2060 (49.93\%) in men and $3762(56.79 \%)$ in women In Ontario, Canada, the most common day surgery procedure was extractions. In the Dental Clinic of the University of Barcelona, $15.34 \%$ of oral surgery treatments were performed on patients younger than 18 years of age. In our study, the number of young patients was significantly lower (7.79\%) because most younger patients were scheduled for surgery under general anaesthesia and were therefore not included in this survey. General anaesthesia represents a risk factor for bacteremia following dental exrtractions (BDE), increasing its prevalence and duration [6]. On the other hand, patients subjected to ambulatory surgery should have a very low risk of serious complications, as they are selected according to their general condition (i.e., the absence of serious systemic diseases) [3].

\section{Conclusions}

Day surgery can be adapted to almost all the oral surgery interventions because these procedures are short-term operations that can be performed with regional anesthesia. Numerous factors contribute to the growing popularity of outpatient surgery;

- Surgery with admission of patients to the hospital costs very much;

- Recent developments at technological advances have led to many outpatient operations;

- The new drugs used under anesthesia allow sedation or general anesthesia with rapid recovery and without side effects;

Table 7 Operation region numbers

\begin{tabular}{lll}
\hline Operation region & Number & $\%$ \\
\hline Posterior & 8161 & 75.92 \\
Anterior & 2418 & 22.49 \\
Posterior and anterior & 137 & 1.27 \\
TMJ & 34 & 0.32 \\
\hline
\end{tabular}

- Day surgery influences less the daily life of patient.

\author{
Abbreviation \\ BDE: Bacteremia following dental extractions \\ Acknowledgements \\ There are no acknowledgements. \\ Funding \\ No funding.
}

Availability of data and materials

No data will be shared due to faculty policies.

\section{Authors' contributions}

All authors read and approved the final version of the manuscript. ABÇ made substantial contributions to conception and design, agreed to be accountable for all aspects of the work in ensuring that questions related to the accuracy or integrity of any part of the work are appropriately investigated and resolved. ÇA made substantial contributions to conception and design, been involved in drafting the manuscript or revising it critically for important intellectual content agreed to be accountable for all aspects of the work in ensuring that questions related to the accuracy or integrity of any part of the work are appropriately investigated and resolved. NK made substantial contributions to conception and design, been involved in drafting the manuscript or revising it critically for important intellectual content, agreed to be accountable for all aspects of the work in ensuring that questions related to the accuracy or integrity of any part of the work are appropriately investigated and resolved. BGK given final approval of the version to be published, agreed to be accountable for all aspects of the work in ensuring that questions related to the accuracy or integrity of any part of the work are appropriately investigated and resolved.

\section{Ethics approval and consent to participate}

Ethics approval and consent to participate was taken from Istanbul University, Oral and Maxillofacial Surgery.

The committee's reference number: 40685465/804.01-569/013.

\section{Consent for publication}

Not applicable.

\section{Competing interests}

The authors declare that they have no competing interests.

\section{Publisher's Note}

Springer Nature remains neutral with regard to jurisdictional claims in published maps and institutional affiliations.

Received: 1 March 2017 Accepted: 22 October 2018

Published online: 30 October 2018

References

1. Daniel A, Haas BD. An update on local anesthetics in dentistry. J Can Dent Assoc. 2002:546-51.

2. Leco Berrocal Ml, Martínez González JM. Social demand in ambulatory Oral surgery. Experience in the master of Oral surgery. Med Oral Patol Oral Cir Bucal. 2008:39-42.

3. Pérez-García S, Chaparro-Avendaño AV, Delgado-Molina E, Berini-Aytés L, Gay-Escoda C. Day case oral surgery in pediatric patients during the year 2000 in the University of Barcelona Dental Clinic (Spain). Med Oral Patol Oral Cir Bucal. 2005:221-30.

4. Syed KB, Zaheer K. Prevalence of impacted molar teeth among Saudi population in the Asir Region, Saudi Arabia_-A retrospective study over 3 years. J Int Oral Health. 2013:43-7.

5. Jena AK, Duggal $R$, Parkash $H$. The distribution of individual tooth impaction in general dental patients of northern India. Community Dent Health. 2010; 27:184-6 Medline: 21046912.

6. Barbosa M, Carmona IT. General anesthesia increases the risk of bacteremia following dental extractions. Oral Surg Oral Med Oral Pathol Oral Radiol Endod. 2010:706-12 